Camila Brasileiro de A. Silva, Virna Ribeiro F. Cestari,

Raquel Sampaio Florêncio, Jair Gomes Linard,

Vera Lucia Mendes de P. Pessoa e Thereza Maria M. Moreira

\title{
FATORES INTERVENIENTES NO ACESSO À CULTURA E LAZER ENTRE ADULTOS JOVENS DE ESCOLAS PÚBLICAS DE UMA CIDADE DO NORDESTE BRASILEIRO
}

\author{
Recebido em: 29/11/2018 \\ Aceito em: 11/09/2019
Samuel Miranda Mattos ${ }^{1}$
Camila Brasileiro de Araújo Silva ${ }^{2}$
Virna Ribeiro Feitosa Cestari ${ }^{3}$
Raquel Sampaio Florêncio ${ }^{4}$
Jair Gomes Linard ${ }^{5}$ \\ Vera Lucia Mendes de Paula Pessoa ${ }^{6}$ \\ Thereza Maria Magalhães Moreira ${ }^{7}$ \\ Universidade Estadual do Ceará \\ Fortaleza - CE - Brasil
}

RESUMO: O objetivo do estudo é analisar os fatores intervenientes ao acesso à cultura e ao lazer entre adultos jovens escolares. Tratou-se de um estudo analítico, realizado com 1.073 adultos jovens matriculados em escolas públicas. Aplicou-se um questionário sobre acesso à cultura e lazer e as variáveis preditoras. Utilizou-se a regressão logística hierarquizada bruta e ajustada, cujas variáveis foram selecionadas pelo método Backward, considerando $\mathrm{p} \leq 0,20$ para entrada no modelo e $\mathrm{p}<0,05$ para permanência. Os resultados apresentaram fatores associados à insatisfação ao acesso à cultura e ao lazer. Identificou os fatores que possam interferir na satisfação desse acesso possibilita planejar e traçar estratégias que assegurem o direito humano.

PALAVRAS CHAVE: Atividades de Lazer. Cultura. Adulto Jovem. Saúde.

\footnotetext{
${ }^{1}$ Professor de Educação Física. Mestrando pelo Programa de Pós-Graduação em Saúde Coletiva da Universidade Estadual do Ceará (UECE).

${ }^{2}$ Professora de Educação Física. Mestre em Saúde Coletiva pela Universidade Estadual do Ceará (UECE).

${ }^{3}$ Enfermeira, Doutoranda pelo Programa de Pós-Graduação em Cuidados Clínico em Enfermagem e Saúde da Universidade Estadual do Ceará (UECE).

${ }^{4}$ Enfermeira. Pós-Doutoranda pelo Programa de Pós-Graduação em Cuidados Clínico em Enfermagem e Saúde da Universidade Estadual do Ceará (UECE). Docente do Programa de Mestrado Profissional em Gestão em Saúde da UECE.

${ }^{5}$ Professor de Educação Física. Mestre em Saúde Coletiva pela Universidade Estadual do Ceará (UECE).

${ }^{6}$ Enfermeira. Doutora em Enfermagem pela Universidade Federal do Ceará (UFC). Docente do Programa de Pós-Graduação em Cuidados Clínicos em Enfermagem e Saúde da Universidade Estadual do Ceará (UECE), Mestrado Profissional da Saúde da Criança e do Adolescente (UECE) e Mestrado Profissional em Transplante de Órgãos (UECE).

${ }^{7}$ Enfermeira e Advogada. Bolsista de Produtividade em Pesquisa do CNPq-nível 1A. Docente da PósGraduação (Mestrado e Doutorado) da UECE nos Programas: 1) Cuidados Clínicos em Enfermagem e Saúde; 2) Saúde Coletiva; 3) Mestrado Profissional Gestão em Saúde. Líder do Grupo de Pesquisa Epidemiologia, Cuidado em Cronicidade e Enfermagem-GRUPECCE-CNPq.
} 


\section{INTERVENING FACTORS IN ACCESS TO CULTURE AND LEISURE AMONG YOUNG ADULTS FROM PUBLIC SCHOOLS IN A CITY IN THE NORTHEAST OF BRAZIL}

ABSTRACT: The objective of this study is to analyze the intervening factors for access to culture and leisure among young adults. This was an analytical study of 1,073 young adults enrolled in public schools. A questionnaire on access to culture and leisure and the predictive variables was applied. The crude and adjusted hierarchical logistic regression was used, whose variables were selected by the Backward method, considering $p \leq 0.20$ for entry into the model and $p<0.05$ for permanence. The results presented factors associated with dissatisfaction with access to culture and leisure. Identified the factors that can interfere in the satisfaction of this access allows to plan and to draw strategies that assure the human right.

KEYWORDS: Leisure Activities. Culture. Young Adult. Health.

\section{Introdução}

A cultura e o lazer, assim como a saúde e educação, representam os maiores desafios para o desenvolvimento de uma nação. Os marcos teóricos que garantem esse direito fundamental está expressos na Declaração Universal dos Direitos Humanos de 1948, na Declaração Universal dos Direitos à Criança de 1959, Constituição Federal de 1988 e no Estatuto da Criança e Adolescente (ECA), sob a lei 8069/90 (MALTA et al., 2014). Para o cumprimento do disposto em legislação, políticas públicas devem ser adotadas, de modo que todo cidadão tenha esse direito assegurado.

O lazer pode ser compreendido como o tempo disponível que o indivíduo possui para além das obrigações diárias e trabalho, sendo uma dimensão da cultura constituída pela expressão humana e caracterizada por um conjunto de ocupações às quais o indivíduo pode entregar-se de livre vontade. Nessa perspectiva, o lazer é um complexo de vivências que se manifesta em diversidade de conteúdo cultural, seja físicoesportivo, sociais, artístico, manuais, intelectual ou turístico (PUKE; MARCELLINO; 2014; ANDRADE et al., 2018). 
Camila Brasileiro de A. Silva, Virna Ribeiro F. Cestari,

Raquel Sampaio Florêncio, Jair Gomes Linard,

Vera Lucia Mendes de P. Pessoa e Thereza Maria M. Moreira

A cultura é um termo polissêmico, pois evocam interesses multidisciplinares,

sendo estudada em áreas como educação física, sociologia, antropologia, história, comunicação, administração, economia, entre outras. Tal realidade concerne ao próprio caráter transversal da cultura, que perpassa diferentes campos da vida cotidiana (CHAPARRO-HURTADO; GUSZMAN-ARIZA, 2014). Neste estudo, o termo cultura não se limita ao processo de desenvolvimento intelectual, mas envolve um conjunto de valores, crenças, costumes, manifestações e hábitos cotidianos praticados em determinada sociedade.

A partir da literatura consultada, observou-se crescente interesse sobre o acesso à cultura e ao lazer entre adultos jovens. $\mathrm{O}$ acesso é relevante por promover diversos benefícios físicos, sociais e psicológicos, contribuindo para melhoria da qualidade de vida (LIZANDRA et al., 2016; ANDRADE et al., 2018). Nesse contexto, torna-se relevante identificar os fatores que intervenientes no acesso à cultura e ao lazer, no intuito de orientar a elaboração de estratégias e implementação de ações que proporcionem a garantia desse direito.

Assim, este estudo teve como objetivo analisar os fatores intervenientes no acesso à cultura e lazer entre adultos jovens escolares.

\section{Método}

Trata-se de estudo analítico, quantitativo, realizado com adultos jovens matriculados em escolas da Secretaria Estadual de Educação do Ceará (Seduc) de ensino regular, situadas em Fortaleza-Ceará-Brasil.

Considerando que a prevalência do acesso à cultura e lazer de adultos jovens é desconhecida, para o cálculo do tamanho amostral foram utilizados os seguintes 
Camila Brasileiro de A. Silva, Virna Ribeiro F. Cestari,

Raquel Sampaio Florêncio, Jair Gomes Linard,

Vera Lucia Mendes de P. Pessoa e Thereza Maria M. Moreira

parâmetros: prevalência estimada em 50\%, margem de erro de 3\% e intervalo de $95 \%$ de confiança (IC95\%), totalizando uma amostra de conveniência selecionadas a partir de 51 escolas escolhidas aleatoriamente, ficando ao final 1.073 adultos jovens escolares pesquisados.

A coleta de dados deu-se por meio da aplicação de um questionário contendo informações relacionadas ao acesso à cultura e lazer e as variáveis preditoras, distribuídas em três blocos: 1) sociodemográficas e econômicas, 2) características de saúde e 3) comportamentais. $\mathrm{O}$ acesso à cultura e lazer foi baseado em nove perguntas: 1) Frequenta a biblioteca da sua escola? 2) Participa de programa de atividade física?; 3) Vai ao cinema e teatro?; 4) Participa de grupos de igreja?; 5) Participa de grupos de teatro?; 6) Participa de grupos de estudo?; 7) Participa de grupos de dança?; 8) Participa de atividades do conselho de saúde?; e 9) Participa do grêmio estudantil da escola?.

Para analisar o acesso à cultura e lazer foi realizado o teste da normalidade, contendo p-valor significativo, com ponto de corte baseado na média. Dessa forma, foi considerado acesso satisfatório os adultos jovens com resposta positiva em três ou mais perguntas e acesso insatisfatório aqueles com resposta negativa em menos de três perguntas. O ponto de corte foi identificado pela verificação da média do acesso.

$\mathrm{Na}$ análise dos dados utilizou-se a regressão logística binária bruta e ajustada para o estudo da associação do acesso à cultura e lazer com os blocos das variáveis preditoras, adotando a categoria acesso insatisfatório como referência, sendo os resultados apresentados pelo cálculo da razão de chances (Odds Ratio - OR), e IC95\%.

No modelo de regressão logística binomial ajustada foi utilizado o método Backward para a seleção das variáveis hierárquica, considerando os três blocos dos 
Camila Brasileiro de A. Silva, Virna Ribeiro F. Cestari,

Raquel Sampaio Florêncio, Jair Gomes Linard,

Vera Lucia Mendes de P. Pessoa e Thereza Maria M. Moreira

fatores, associados considerando $p$-valor $\leq 0,20$ para entrada no modelo e $p<0,05$ para permanência.

Este projeto foi aprovado pelo comitê de ética em pesquisa da Universidade Estadual do Ceará, sob o protocolo de número 484894/2012-9 e financiado pelo Conselho Nacional de Desenvolvimento Científico e Tecnológico- CNPq, Edital Universal 14/2012, tendo a pesquisa seguido todos os princípios éticos e legais em todas as fases do estudo.

\section{Resultados}

Os resultados obtidos com base na análise binária ajustada do acesso à cultura e lazer, utilizando a categoria insatisfatória como referência, foram dispostos na Tabela 1. Observou-se que nos jovens com filhos aumentou em 50\% a chance de ter acesso à cultura e lazer insatisfatório $[\mathrm{OR}=1,561$ (IC95\%=1,066-2,286)]. Em relação às características de saúde, apenas o estado nutricional permaneceu no estudo. Constatouse que os adultos jovens que possuíam peso normal apresentaram 37,6\% menos chances de ter acesso insatisfatório do que aqueles com excesso ponderal [OR=0,624 $(\mathrm{IC} 95 \%=0,430-0,905)]$.

As variáveis relacionadas ao comportamento apresentaram-se mais associadas ao acesso à cultura e lazer, permanecendo no modelo final o etilismo, comportamento sedentário no fim de semana e ter tentado perder peso. Em relação ao etilismo, os jovens em uso de álcool apresentaram $28,7 \%$ de chances reduzidas para o acesso à cultura e lazer em relação aos que não fazem uso de álcool $[\mathrm{OR}=0,713$ (IC95\%= 0,5140,989)]. 
Camila Brasileiro de A. Silva, Virna Ribeiro F. Cestari,

Raquel Sampaio Florêncio, Jair Gomes Linard,

Vera Lucia Mendes de P. Pessoa e Thereza Maria M. Moreira

Verificou-se que adultos jovens com comportamento sedentário no fim de semana, apresentaram $44,2 \%$ mais chances ao acesso insatisfatório sobre aqueles sem comportamento sedentário $[\mathrm{OR}=1,442 \quad(\mathrm{IC} 95 \%=1,024-2,030)]$. Os jovens que já tentaram perder peso possuíram 31,1\% menos chances de terem acesso insatisfatório do que os que nunca tentaram perder peso [OR=0,689 (IC95\%=0,478-0,999)].

Tabela 1: Análise multivariada dos fatores intervenientes do acesso à cultura e lazer por adultos jovens escolares; Fortaleza; Ceará. Brasil, 2016.

\begin{tabular}{|c|c|c|c|c|}
\hline Blocos & $\begin{array}{c}\text { OR BRUTA } \\
(\text { IC95\%) }\end{array}$ & p-valor & $\begin{array}{c}\text { OR AJUST } \\
\text { (IC95\%) }\end{array}$ & p-valor \\
\hline \multicolumn{5}{|c|}{ Bloco 1: Variáveis sociodemográficas e econômicas } \\
\hline Sexo & & 0,554 & & - \\
\hline Masculino & 1 & & - & \\
\hline Feminino & $1,090(0,820-1,448)$ & & - & \\
\hline Situação conjugal & & 0,679 & & - \\
\hline Com companheiro & $1,077(0,759-1,527)$ & & - & \\
\hline Sem companheiro & 1 & & - & \\
\hline Possui Filhos & & 0,001 & & 0,022 \\
\hline Sim & $1,751(1,259-2,433)$ & & $1,561(1,066-2,286)$ & \\
\hline Não & 1 & & 1 & \\
\hline Recebe bolsa família & & 0,062 & & 1,114 \\
\hline Sim & $0,739(0,538-1,016)$ & & $0,753(0,530-1,070)$ & \\
\hline Não & 1 & & 1 & \\
\hline Possui casa própria & & 0,871 & & \\
\hline $\operatorname{Sim}$ & $0,871(0,649-1,170)$ & & - & - \\
\hline Não & 1 & & - & \\
\hline Idade & & 0,378 & & \\
\hline 21 & $0,683(0,436-1,071)$ & & - & \\
\hline 22 & $0,854(0,512-1,425)$ & & - & - \\
\hline 23 & $0,615(0,341-1,110)$ & & - & \\
\hline 24 & 1 & & & \\
\hline Renda Individual & & 0,375 & & 0,174 \\
\hline Sem renda & $1,328(0,872-2,023)$ & & $1,557(0,976-2,485)$ & \\
\hline$<1$ salário & $1,297(0,864-1,947)$ & & $1,386(0,891-2,156)$ & \\
\hline$\geq 1$ salário & 1 & & 1 & \\
\hline Mora sozinho & & 0,558 & & - \\
\hline Sim & $1,309(0,532-3,219)$ & & - & \\
\hline Não & 1 & & - & \\
\hline Ocupação & & 0,187 & & - \\
\hline Trabalha & $0,959(0,710-1,296)$ & & - & \\
\hline Estuda & $0,499(0,236-1,055)$ & & - & \\
\hline Só estuda & 1 & & - & \\
\hline \multicolumn{5}{|c|}{ Bloco 2: Características de Saúde } \\
\hline $\begin{array}{l}\text { Tem problema de } \\
\text { saúde }\end{array}$ & & 0,348 & & - \\
\hline Sim & 1 & & - & \\
\hline Não & $1,168(0,844-1,616)$ & & - & \\
\hline $\begin{array}{l}\text { Autopercepção de } \\
\text { saúde }\end{array}$ & & 0,012 & & 0,294 \\
\hline Positiva & $0,688(0,514-0,919)$ & & $0,840(0,606-1,163)$ & \\
\hline Negativa & 1 & & 1 & \\
\hline
\end{tabular}


Camila Brasileiro de A. Silva, Virna Ribeiro F. Cestari,

Raquel Sampaio Florêncio, Jair Gomes Linard,

Vera Lucia Mendes de P. Pessoa e Thereza Maria M. Moreira

\begin{tabular}{|c|c|c|c|c|}
\hline \multicolumn{2}{|l|}{$\begin{array}{l}\text { Acha que está acima } \\
\text { do peso }\end{array}$} & \multicolumn{2}{|l|}{0,122} & \multirow[t]{2}{*}{-} \\
\hline Sim & $1,268(0,938-1,714)$ & & - & \\
\hline Não & 1 & & - & \\
\hline Estado Nutricional & & 0,010 & & 0,013 \\
\hline Normal & $0,682(0,509-0,913)$ & & $0,624(0,430-0,905)$ & \\
\hline Excesso Ponderal & 1 & & 1 & \\
\hline \multicolumn{5}{|c|}{ Bloco 3: Variáveis comportamentais } \\
\hline Uso de cigarro & & 0,056 & & - \\
\hline Sim & 1 & & - & \\
\hline Não & $1,770(0,986-3,176)$ & & - & \\
\hline Etilismo & & 0,092 & & 0,043 \\
\hline Sim & 1 & & 1 & \\
\hline Não & $1,292(0,959-1,741)$ & & $0,713(0,514-0,989)$ & \\
\hline \multicolumn{2}{|c|}{ Comportamento Sedentário na semana } & 0,907 & & - \\
\hline Sem CS & 1 & & - & \\
\hline Com CS & $1,018(0,749-1,384)$ & & - & \\
\hline \multicolumn{2}{|c|}{ Comportamento sedentário no fim de semana } & 0,031 & & 0,036 \\
\hline Sem CS & 1 & & 1 & \\
\hline Com CS & $0,715(0,526-0,970)$ & & $1,442(1,024-2,030)$ & \\
\hline \multicolumn{2}{|c|}{ Recomendação de prática de Atividade Física } & 0,196 & & - \\
\hline Sim & $1,210(0,907-1,614)$ & & - & \\
\hline Não & 1 & & - & \\
\hline \multicolumn{2}{|l|}{ Tentou perder peso } & 0,241 & & 0,045 \\
\hline Sim & $0,841(0,629-1,123)$ & & $0,689(0,478-0,999)$ & \\
\hline Não & 1 & & 1 & \\
\hline \multicolumn{2}{|l|}{$\begin{array}{l}\text { Deslocamento para a } \\
\text { escola }\end{array}$} & 0,873 & & \\
\hline Ativo & $1,026(0,748-1,408)$ & & - & - \\
\hline Passivo & 1 & & - & \\
\hline \multicolumn{2}{|l|}{ Evasão Escolar } & 0,004 & & 0,062 \\
\hline Sim & $1,671(1,181-2,364)$ & & $1,452(0,982-2,146)$ & \\
\hline Não & 1 & & 1 & \\
\hline
\end{tabular}

\section{Discussão}

Considerando que o acesso à cultura e espaços de lazer influencia na qualidade de vida do ser humano, esse estudo buscou analisar os fatores intervenientes neles. A partir dos resultados apresentados, verificou-se que o acesso insatisfatório à cultura e lazer entre adultos jovens esteve associada à presença de filhos, sobrepeso, etilismo, comportamento sedentário e evasão escolar.

$\mathrm{Na}$ análise estratificada das variáveis sociodemográficas e econômicas, os adultos jovens com filhos mostraram ter 1,56 maior chance de apresentar acesso à cultura e lazer insatisfatório $(\mathrm{p}=0,022)$. Esse achado é comum em grupos com essa 
Camila Brasileiro de A. Silva, Virna Ribeiro F. Cestari,

Raquel Sampaio Florêncio, Jair Gomes Linard,

Vera Lucia Mendes de P. Pessoa e Thereza Maria M. Moreira

característica, pois se sabe que os pais demandam maior alocação do tempo para o cuidado dos filhos, o que os fazem negligenciar seu tempo de lazer (BARBOSA, 2018).

Acredita-se que os pais acabam acompanhando seus filhos nas atividades de lazer destes, que é caracterizada, em grande parte, ao hábito de assistir TV (FERRARI et al., 2019). Um estudo que avaliou a díade pais-filhos em relação visualização da TV mostrou que um percentual significativo excedeu o limiar de duas horas nos dias úteis e finais de semana (Jago, R.; Solomon-Moore, E.; Macdonald-Wallis, C. et al., 2017).

Assistir à TV é uma das atividades que consomem mais tempo do ser humano e pode trazer diversos riscos para saúde. Frequentemente, esse hábito está relacionado ao consumo alimentar em demasia e de baixo valor nutritivo. Em pesquisa para analisar a evolução da frequência do hábito de assistir à TV no Brasil e identificar a associação entre esse hábito e o consumo alimentar, os autores verificaram que adultos jovens assistem à TV por ao menos 3 horas por dia, sendo este hábito associado ao menor consumo de alimentos saudáveis (MATA et al., 2016).

No que se refere ao estado nutricional dos jovens adultos, essa foi uma característica de saúde que apresentou relação com o acesso à cultura e lazer neste estudo. Aqueles com peso normal tiveram 0,62 menor chance de acesso insatisfatório $(p=0,013)$. Além disso, a tentativa de perder peso diminuiu em $31 \%$ a chance dos jovens terem acesso ao lazer.

A prevalência do excesso ponderal tem aumentado consideravelmente nos últimos anos. O Brasil está em estágio avançado de transição nutricional, com altas taxas de sobrepeso e baixas taxas de baixo peso (BISPO et al., 2015). Em adultos jovens, o estado nutricional alterado está associado com estilo de vida ruim, a comportamento sedentário, obesidade, diabetes mellitus tipo II, doenças 
Camila Brasileiro de A. Silva, Virna Ribeiro F. Cestari,

Raquel Sampaio Florêncio, Jair Gomes Linard,

Vera Lucia Mendes de P. Pessoa e Thereza Maria M. Moreira

cardiopulmonares e câncer (O’DONOGHUE et al., 2016; SILVA; FERREIRA;

SEGHETO et al., 2016).

Nesse escopo, o estilo de vida atual tem sido considerado uma das principais razões para o aumento da prevalência do excesso de peso. Reitera-se que aspectos físicos, econômicos, sociais e culturais do ambiente podem incentivar o equilíbrio de energia positiva, promovendo a obesidade (SIQUEIRA et al., 2015). Isso pode ser explicado pela dificuldade de acesso aos ambientes de lazer que diminuem a prática de atividade física.

Uma possível explicação para os adultos jovens deste estudo apresentarem excesso ponderal é devido à presença do comportamento sedentário no tempo de lazer.

Salienta-se que os resultados também evidenciaram que o acesso à cultura e lazer foi insatisfatório para aqueles indivíduos com comportamento sedentário no final de semana. Verificou-se que os adultos jovens que apresentaram esse comportamento tiveram 1,44 maior chance de não ter o acesso $(p=0,036)$.

O comportamento sedentário representa atividades de pequena movimentação que ocorrem com o corpo na posição sentada ou reclinada, e têm gasto energético próximo ao observado no estado de repouso, ou seja, $\leq 1,5$ unidades do equivalente metabólico (MET). Trata-se de um comportamento distinto da atividade física, não mais caracterizado pela ausência desta. Portanto, vê-se que muitas pessoas que praticam atividade física podem possuir comportamento sedentário, com fatores correlatos e determinantes e implicações para saúde próprios (GUERRA; FARIAS JÚNIOR; FLORINDO, 2016). 
Camila Brasileiro de A. Silva, Virna Ribeiro F. Cestari,

Raquel Sampaio Florêncio, Jair Gomes Linard,

Vera Lucia Mendes de P. Pessoa e Thereza Maria M. Moreira

O estudo do comportamento sedentário tem sido nos últimos dez anos reconhecimento como uma questão de saúde pública e investigações apontam sua relação com efeitos deletérios para saúde (MENEGUCI et al., 2015).

A prática de exercício físico nas atividades de lazer reduz a incidência de doenças crônicas (RICHARD et al., 2015). Assim, destaca-se a importância da implementação de programas educativos no intuito de maior adesão às práticas saudáveis.

Publicações recentes têm demonstrado que a proporção de adultos que praticam atividade física no lazer por, pelo menos, 30 minutos por dia em cinco ou mais dias na semana aumentou de 12,8\% em 2006 para 14,9\% em 2012. Ademais, evidenciam uma redução do deslocamento ativo para o trabalho e na proporção de adultos que assistem televisão por mais de três horas por dia (MIELKE et al., 2014; MIELKE et al., 2015).

Neste estudo, também foi verificada a relação entre álcool e acesso, onde o uso de álcool configurou-se como fator interveniente do acesso à cultura e lazer (OR ajustado: 0,71; $\mathrm{p}=0,043)$. $\mathrm{O}$ uso de álcool, do tabaco e de outras drogas são comportamentos de risco que se iniciam, geralmente, em idades precoces e se estendem por toda a vida. Ademais, associam-se a maior número de faltas e evasão escolar, distúrbios alimentares e percepção de saúde mais negativa (TESTON et al., 2016).

O uso de substância lícitas e ilícitas tem se configurado como uma preferência entre jovens e adultos jovens como atividade de lazer, acarretando inúmeras complicações para a saúde, como alterações somáticas e psiquiátrica, bem como consequências econômicas e sociais. Dentre os fatores de risco para o consumo de bebidas alcoólicas, destaca-se o comportamento sedentário (EJSING et al., 2015). 
Camila Brasileiro de A. Silva, Virna Ribeiro F. Cestari,

Raquel Sampaio Florêncio, Jair Gomes Linard,

Vera Lucia Mendes de P. Pessoa e Thereza Maria M. Moreira

Pesquisa desenvolvida em Pernambuco buscou conhecer os significados do uso de álcool entre adultos jovens. Dentre as diversidades de tramas que os marcadores sociais de diferenciação engendram, os significados atribuídos ao uso de álcool foram relacionados, principalmente, às poucas atividades de lazer e relações de gênero (SILVA; MENEZES, 2016).

Em outro estudo, verificou-se a associação entre exposição ao consumo de bebidas alcoólicas e acesso ao lazer em 4.207 jovens de uma rede pública estadual. Os autores observaram que os jovens que consumiram bebidas alcoólicas tinham chance $27 \%$ inferior de acesso satisfatório ao lazer quando comparados aos que não consumiam (BEZERRA et al., 2015), corroborando com o estudo atual.

\section{Conclusão}

O acesso à cultura e ao lazer é um processo de trocas simbólicas com variadas expressões na sociedade. No contexto dos adultos jovens escolares, identificar os fatores que possam interferir na satisfação desse acesso possibilita planejar e traçar estratégias que assegurem o direito humano.

Os fatores associados à dificuldade no acesso ao lazer e à cultura entre os adultos jovens que participaram deste estudo foram possuir filho, estado nutricional alterado, etilismo, comportamento sedentário no fim de semana e tentou perder peso alguma vez.

O acesso ao lazer e à cultura é afetada por características da saúde e comportamentais como evidenciado nos resultados e discussão. No entanto, é preciso destacar que para esse acesso se concretizar devem existir políticas mais efetivas de 
Camila Brasileiro de A. Silva, Virna Ribeiro F. Cestari,

Raquel Sampaio Florêncio, Jair Gomes Linard,

Vera Lucia Mendes de P. Pessoa e Thereza Maria M. Moreira

promoção da saúde que repercutam na construção de ambientes saudáveis e adequados

para a prática do lazer.

Os resultados ratificam a necessidade de práticas educativas em saúde no ambiente escolar, uma vez que a promoção da saúde destaca-se como uma ação de corresponsabilização social e pode contribuir com o desenvolvimento de projetos que repercutam positivamente na vida e bem-estar de indivíduo e coletividade. Contudo, a educação é um referencial para a construção de conhecimentos e desenvolvimento de valões e atitudes em prol de uma sociedade mais conscientizada.

Reitera-se, ainda, a relevância de investimentos e interações entre profissionais da saúde, pais, alunos e gestão escolar, no intuito de compor redes de compromisso, estimulando debates e ações a partir da realidade da comunidade escolar e sociedade.

\section{REFERÊNCIAS}

ANDRADE, R.D. et al. Validade de construto e consistência interna da Escala de Práticas no Lazer (EPL) para adultos. Cien Saude Coletiva. v. 23, n.2, p. 519-28, 2018 .

BARBOSA, A.L.N.H. Tendências na alocação do tempo no Brasil: trabalho e lazer. Revista Brasileira de Estudos de População, v. 3, 5(1), p 1-28, 2018. https://doi.org/10.20947/s102-3098a0063

BEZERRA, J. et al. Consumo de bebidas alcoólicas e tabagismo: associação com inatividade física no lazer e comportamento sedentário. Rev Andal Med Deporte. v. 8, n. 1, p 1-6, 2015.

BISPO, S. et al. Nutritional status of urban adolescentes: individual, household and neighborhood factors based on data from The BH Health Study. Cad Saude Pública. v. 3, n.1, p. 232-45, 2015.

CHAPARRO-HURTADO, H.R.; GUZMÁN-ARIZA, C.M. Los (múltiples) centros de la esfera: cultura, juventude y educación como aventuras contemporâneas. Rev Latinoam Cienc Soc Niñez Juv. v. 12, n. 2, p 691-701, 2014. 
Camila Brasileiro de A. Silva, Virna Ribeiro F. Cestari,

Raquel Sampaio Florêncio, Jair Gomes Linard,

Vera Lucia Mendes de P. Pessoa e Thereza Maria M. Moreira

EJSING, L.K.; BECKER, U.; TOLSTRUP, J.S.; FLENSBORD-MADSEN, T. Physical activity and risk of alcohol use disorders: results from a prospective cohort study. Alcohol Alcohol. v. 50, n. 2, p 206-12, 2015.

FERRARI G.L, PIRES C, SOLÉ D, MATSUDO V, KATZMARZYK P.T, FISBERG M. Factors associated with objectively measured total sedentary time and screen time in children aged 9-11 years. J Pediatr (Rio J). v. 95, p. 94-105, 2019.

GUERRA, P.H.; FARIAS JÚNIOR, J.C.; FLORINDO, A.A. Comportamento sedentário em crianças e adolescentes brasileiros: revisão sistemática. Rev Saude Pública. v. 50, n.1, p. 1-15, 2016.

JAGO, R., SOLOMON-MOORE, E., MACDONALD-WALLIS, C. et al. Association of parents' and children's physical activity and sedentary time in Year 4 (8-9) and change between Year 1 (5-6) and Year 4: a longitudinal study. Int J Behav Nutr Phys Act. 14, 110 (2017).

LIZANDRA, J.; DEVÍS-DEVÍS, J.; PÉREZ-GIMENO, E.; VALENCIA-PERIS, A.; PEIRÓ-VELERT, C. Does sedentary behavior predict academic performance in adolescentes or the other way round? A longitudinal path analysis. PLoS One. v. 11, n. 4, p 1-13, 2016.

MALTA, D.C. et al. A implementação das prioridades da Política Nacional de Promoção da saúde, um balanço, 2006 a 2014. Ciênc Saude Coletiva. v. 19, n. 11, p. 4301-12, 2014.

MATA, E.G. et al. Hábito de assistir à televisão e sua relação com a alimentação: resultados do período de 2006 a 2014 em capitais brasileiras. Cad Saude Pública. v. 32, n. 9, 2016.

MENEGUCI, J. et al. Comportamento sedentário: conceito, implicações fisiológicas e os procedimentos de avaliação. Motricidade. v. 11, n. 1, p. 160-74, 2015.

MIELKE, G.I. et al. Times trends of physical activity and sedentary behaviour in state capital of North region of Brazil: 2006 - 2013. Rev Bras Ativ Fis Saúde. v. 20, n. 2, p $.130-40,2015$.

MIELKE, G.I. et al. Times trends of physical activity and television viewing time in Brazil: 2006 - 2012. Int J Nutr Phys Act. v. 11, n. 101, 2014.

O'DONOGHUE, G. et al. A systematic review of correlates of sedentary behaviour in adults aged 18 - 65 years: a sócio-ecological approach. BMC Public Health. v.16, n. $163,2016$.

PUKE, N.; MARCELLINO, N.C. Possibilidades de interface entre lazer e fenomenologia. Movimento. v. 20, n. 1, p. 307-27, 2014.

RICHARD, A. et al. Effects of leisure-time and occupational physical activity on total mortality risk in NHANES III according sex, ethnicity, central obesity and age. J Phys Act Health. v. 12, n. 2, p. 184-92, 2015. 
Camila Brasileiro de A. Silva, Virna Ribeiro F. Cestari,

Raquel Sampaio Florêncio, Jair Gomes Linard,

Vera Lucia Mendes de P. Pessoa e Thereza Maria M. Moreira

SILVA, R.A.; MENEZES, J.A.; Reflexões sobre o uso de álcool entre jovens quilombolas. Psicol Soc. v. 28, n. 1, p. 84-93, 2016.

SILVA, R.R.A.; FERREIRA, F.G.; SEGHETO, W. Atividade física no lazer, estado nutricional autoreferido e tempo gasto sentado em trabalhadores do comércio. Rev Bras Nutr Esportiva. v.10. n. 56, p 222-9, 2016.

SIQUEIRA, K. et al. Interrelationships between nursing workers' state of nutrition, socio demographic factors, work and health habits. Ciênc Saude Coletiva. v. 20, n .6, p. 1925-35, 2015.

TESTON, E.F. et al. Fatores associados às doenças cardiovasculares em adultos. Medicina. v. 49, n. 2, p. 95-102, 2016.

\section{Endereço dos Autores:}

Samuel Miranda Mattos

Av. Silas Munguba, 1700, Itaperi

Fortaleza, CE - 60.740-000

Endereço Eletrônico: profsamuelmattos@gmail.com

Camila Brasileiro de Araújo Silva

Av. Silas Munguba, 1700, Itaperi

Fortaleza, CE - 60.740-000

Endereço Eletrônico: camilabrasileiro19@gmail.com

Virna Ribeiro Feitosa Cestari

Av. Silas Munguba, 1700, Itaperi

Fortaleza, CE - 60.740-000

Endereço Eletrônico: virna.ribeiro@hotmail.com

Raquel Sampaio Florêncio

Av. Silas Munguba, 1700, Itaperi

Fortaleza, CE - 60.740-000

Endereço Eletrônico: raquelsampy@hotmail.com

Jair Gomes Linard

Av. Silas Munguba, 1700, Itaperi

Fortaleza, CE - 60.740-000

Endereço Eletrônico: jlinard45@gmail.com

Vera Lucia Mendes de Paula Pessoa

Av. Silas Munguba, 1700, Itaperi

Fortaleza, CE - 60.740-000

Endereço Eletrônico: pessoa_vera@hotmail.com

Thereza Maria Magalhães Moreira 
Samuel Miranda Mattos,

Fatores Intervenientes no Acesso à Cultura e Lazer...

Camila Brasileiro de A. Silva, Virna Ribeiro F. Cestari,

Raquel Sampaio Florêncio, Jair Gomes Linard,

Vera Lucia Mendes de P. Pessoa e Thereza Maria M. Moreira

Av. Silas Munguba, 1700, Itaperi

Fortaleza, CE - 60.740-000

Endereço Eletrônico: tmmmoreira@pq.cnpq.br 